\title{
Detection of testosterone administration based on the carbon isotope ratio profiling of endogenous steroids: international reference populations of professional soccer players
}

\author{
E Strahm, ${ }^{1}$ C Emery, ${ }^{1}$ M Saugy, ${ }^{1}$ J Dvorak, ${ }^{2}$ C Saudan'
}

${ }^{1}$ Swiss Laboratory for Doping Analyses, University Center of Legal Medicine, West Switzerland, Epalinges, Switzerland; ${ }^{2}$ FIFA Medical Assessment and Research Centre, Zürich, Switzerland

Correspondence to: C Saudan, Swiss Laboratory for Doping Analyses, University Center of Legal Medicine, West Switzerland, Chemin des Croisettes 22, 1066 Epalinges, Switzerland; Christophe. Saudan@chuv.ch

Accepted 1 June 2009 Published Online First 22 June 2009

\section{UNLOCKI}

This paper is freely available online under the BMJ Journals unlocked scheme, see http:// bjsm.bmj.com/info/unlocked.dtl

\begin{abstract}
Background and objectives: The determination of the carbon isotope ratio in androgen metabolites has been previously shown to be a reliable, direct method to detect testosterone misuse in the context of antidoping testing. Here, the variability in the ${ }^{13} \mathrm{C} /{ }^{12} \mathrm{C}$ ratios in urinary steroids in a widely heterogeneous cohort of professional soccer players residing in different countries (Argentina, Italy, Japan, South Africa, Switzerland and Uganda) is examined.
\end{abstract}

Methods: Carbon isotope ratios of selected androgens in urine specimens were determined using gas chromatography/combustion/isotope ratio mass spectrometry (GCC-IRMS).

Results: Urinary steroids in Italian and Swiss populations were found to be enriched in ${ }^{13} \mathrm{C}$ relative to other groups, reflecting higher consumption of C3 plants in these two countries. Importantly, detection criteria based on the difference in the carbon isotope ratio of androsterone and pregnanediol for each population were found to be well below the established threshold value for positive cases. Conclusions: The results obtained with the tested diet groups highlight the importance of adapting the criteria if one wishes to increase the sensitivity of exogenous testosterone detection. In addition, confirmatory tests might be rendered more efficient by combining isotope ratio mass spectrometry with refined interpretation criteria for positivity and subject-based profiling of steroids.

Anabolic androgenic steroids (AAS), particularly testosterone, are commonly used by athletes to enhance sports performance. The extent of misuse of AAS by competing athletes is clearly shown by the statistics of the adverse analytical findings reported by accredited laboratories in the last few years. Moreover, the same hormones that are included in the list of prohibited substances published by the World Anti-Doping Agency (WADA) are also misused by young people and non-competing amateurs. ${ }^{1}$ Chronic administration of AAS has been shown to produce endocrine, somatic and neuropsychiatric side effects. Psychological side effects are of particular concern because they induce violent behaviours with potentially serious consequences for the society at large. ${ }^{1}$

Testosterone doping cannot be detected by simply measuring the levels of endogenous hormones such as androgens in biological fluids. The variability in human metabolism of this compound is simply too large. ${ }^{2}$ In the antidoping field evidence of testosterone administration relies on a confirmatory procedure that uses isotope ratio mass spectrometry (IRMS). ${ }^{3}$ A technical document established by WADA in 2004, stipulates that a urine sample must be analysed by IRMS to determine the carbon isotope ratios of androgens if the peak area ratio of testosterone/epitestosterone equivalent to the glucuronide is equal to or greater than 4.0 or if altered steroid profiles are determined. ${ }^{4}$ Endogenous testosterone is produced in the human body via cholesterol metabolism..$^{56}$ Detection of testosterone doping relies on the general observation that endogenous testosterone has a different ${ }^{13} \mathrm{C}$ content compared to hemisynthetic testosterone used in pharmaceutical preparations. Such detection is possible because the carbon atoms in steroid molecules originate primarily in atmospheric $\mathrm{CO}_{2}$, which is fixed through photosynthesis. The most important photosynthetic pathways used by plants are the so-called C3 and C4 pathways. ${ }^{78}$ Significant isotope fractionation occurs during photosynthetic carbon fixation, depending on the mode of $\mathrm{CO}_{2}$ fixation. Thus, the key enzymes that fix $\mathrm{CO}_{2}$ in $\mathrm{C} 3$ plants discriminate more strongly against ${ }^{13} \mathrm{CO}_{2}$ than their analogues in C4 plants. As a result, the two types of plants differ by about $14 \%$ in the isotopic composition of their tissues. The natural abundance carbon isotopic ratio is expressed as a $\delta$ value relative to an international standard (Vienna Pee Dee Belemnite, VPDB; equation 1):

$\delta^{13} \mathrm{C} \%=\frac{\left({ }^{13} \mathrm{C} / /^{12} \mathrm{C}\right)_{\text {sample }}-\left({ }^{13} \mathrm{C} /{ }^{12} \mathrm{C}\right)_{\text {standard }}}{\left({ }^{13} \mathrm{C} /{ }^{12} \mathrm{C}\right)_{\text {standard }}} \times 1000$

The practical outcome is the fact that the distribution of carbon isotopes in an animal reflects the relative abundance of food in the diet that originates directly or indirectly from C4 and C3 plants (either plants or animals that are lower in the food chain). Controlled diet studies have shown that the isotopic composition of the whole body of an animal is enriched by about $1 \%$ as a function of the isotopic composition of its diet. ${ }^{9}$

Although the diet composition of an athlete has a predominant influence on the carbon isotope ratio of the steroids excreted in urine, there have been no published comparisons of this diagnostic parameter in any cohort of elite athletes in a specific sports category. In this study, the range of 
the carbon isotope ratio of the steroids relevant to antidoping analysis was investigated in urine specimens obtained from toplevel soccer player populations residing in six countries, namely Argentina, Italy, Japan, Republic of South Africa, Switzerland and Uganda. The determination of threshold values specific for a given diet and athlete metabolism is expected to significantly improve the detection of testosterone misuse by means of stable isotope methodology.

\section{METHODS}

\section{Population and sample collection}

Urine samples were collected from 171 male soccer players aged from 18 to 36 (mean (SD) age 24.5 (3.8) years old). These elite athletes were competing in top-level teams in championships taking place in 6 different countries: Argentina (ARG, $\mathrm{n}=31$ ), Italy (ITA, $n=19)$, Japan (JAP, $n=32$ ), The Republic of South Africa (SAF, $\mathrm{n}=30$ ), Switzerland (SWI, $\mathrm{n}=31$ ) and Uganda (UGA, $n=28$ ). The study was initiated after validation of the protocol by the ethical committee of the University of Lausanne, Switzerland. A medical officer informed the participants in each soccer team about the goal of the project and the period of sampling. The subjects were not asked for a detailed description of their eating habits, but they were asked to sign a consent form which stipulated that they did not take any medicine that could influence their steroid profile for at least 4 weeks prior to urine collection. The urine samples were collected by the medical officer using BEREG-KITs (Berlinger AG, Ganterschwil, Switzerland). The anonymised samples were sent to the laboratory and the temperature was maintained at $4^{\circ} \mathrm{C}$. Upon arrival, the samples were distributed in $20-\mathrm{ml}$ glass containers and frozen at $-20^{\circ} \mathrm{C}$ prior to extraction. The information made available to the laboratory included age, sex, country of residence, as well as the date and time of urine collection.

\section{Analytical methods}

To reasonably ensure that no athlete used a doping agent prior to urine collection, all specimens were initially subjected to the analytical assays carried out in our WADA-accredited laboratory for the screening of substances prohibited in-competition. ${ }^{10}$

For the purpose of our study, a gas chromatography/ combustion/isotope ratio mass spectrometry (GC-C-IRMS) method was used to determine the carbon isotope ratio of androsterone (A), etiocholanolone (Etio), 16(5 $\alpha$ )-androstenol $(16 \mathrm{EN})$ and $5 \beta$-pregnanediol (PD). ${ }^{11}$ This assay, based on three solid phase extraction (SPE) cleanup steps, required an initial volume of urine of $10 \mathrm{ml}$. The urine samples used for the entire study were processed in different batches $(n=7)$ and included negative quality controls ( $\mathrm{OC}$ ). It is noteworthy that no drift was observed in the carbon isotope ratio of the analytes in the QC during the period of analysis ( 5 months) and the standard deviations reached $0.39 \%$, $0.34 \%$, $0.34 \%$ and $0.29 \%$ for Etio, A, $16 \mathrm{EN}$ and $\mathrm{PD}$, respectively.

\section{Data analysis}

All statistical analyses were performed using S-PLUS V. 7.0 (TIBCO Software Inc, Palo Alto, California, USA) for Windows. For distribution testing, the Kolmogorov-Smirnov test of normality was used. Testing of statistical differences among two normally distributed groups relied on the two-sample t test. A one-way analysis of variance (ANOVA) was applied to compare more than two groups. The level of significance was established at $\mathrm{p}<0.05$ for all statistics.

\section{RESULTS AND DISCUSSION}

The carbon isotope ratios of androsterone and etiocholanolone are usually determined to prove doping with testosterone or a testosterone prohormone, because both compounds originate in the same androgen biosynthetic pathway (fig 1) and are excreted in the urine at relatively high concentrations compared to the other androgens. The sensitivity of IRMS may be the limiting factor in the accurate determination of low-level testosterone metabolites such as $5 \alpha$-androstane- $3 \alpha, 17 \beta$-diol and $5 \beta$-androstane- $3 \alpha, 17 \beta$-diol. The metabolism of an exogenous anabolic steroid in humans will cause depletion in ${ }^{13} \mathrm{C}$ of the steroid itself or of its metabolites in urine specimens, since the primary source of synthetic preparations are phytosterols extracted from ${ }^{13} \mathrm{C}$ depleted plants (C3 plants), mainly soy and Mexican yam. During the drug washout period, the ${ }^{13} \mathrm{C} /{ }^{12} \mathrm{C}$ ratio of these compounds will progressively return to the natural background values characteristic of the diet of the individual. Ultimately, the variation in the ${ }^{13} \mathrm{C} /{ }^{12} \mathrm{C}$ ratio of the testosterone metabolites will depend on ${ }^{13} \mathrm{C}$ depletion of the pharmaceutical preparation administered, as well as the athlete's baseline values and individual metabolism. ${ }^{12}$ In our study, we focused on populations representative of top-level athletes for whom a very low prevalence of doping was assumed. Figure 2 shows the ${ }^{13} \mathrm{C} /{ }^{12} \mathrm{C}$ ratio expressed in $\delta^{13} \mathrm{C}$ values (\%) of androsterone and etiocholanolone together with $16(5 \alpha)$-androstenol and $5 \beta$-pregnanediol. Of note, we did not find any evidence against the assumption that the $\delta^{13} \mathrm{C}$ values were normally distributed for each target steroid. Comparisons between the different groups show that Italian and Swiss populations have more negative $\delta^{13} \mathrm{C}$ values for all the tested steroids. At the same time, the Japanese soccer players exhibited intermediate $\delta^{13} \mathrm{C}$ values compared to those of the European countries and the group comprising ARG, SAF and UGA. For all the studied steroids, Argentinean players and players of African countries displayed a similar enrichment in ${ }^{13} \mathrm{C}(\mathrm{p}>0.2)$.

The $\delta^{13} \mathrm{C}$ values measured for the target compounds in each of the 171 urine specimens ranged from $-17.2 \%$ to $-25.2 \%$, thus reflecting the extent of isotopic fractionation as a function of the athletes' diets and metabolisms. In order to understand these results, it is important to consider the period of time during which isotope ratios of steroids reflect one's individual diet. Although in the case of human steroids, the turnover of stable carbon isotopes is still poorly understood, the mechanism of isotopic equilibration in steroids was nevertheless investigated in a recent preliminary study. ${ }^{13}$ Specifically, changes from standard European eating habits to a C4 plant-dominated diet revealed a common half life of about 17 days in the ${ }^{13} \mathrm{C}$ enrichment process of the urinary steroids. In our study, we can assume that no significant shifts in the eating habits of the athletes occurred in the month prior to urine collection. Thus, it may be hypothesised that the excreted steroids were isotopically equilibrated with respect to the athlete's diet. As stated previously, the distribution of carbon isotopes in animals reflects the relative abundance of $\mathrm{C} 4$ and $\mathrm{C} 3$ plants in their diet or those of the animals that are lower in the food chain. Compounds from C3 plants (eg, wheat, barley, oats, sugar beet, rye, cotton) generally have carbon isotopic values in the $-35 \%$ to $-22 \%$ range whereas compounds from C4 plants (eg, corn, millet, sugar cane, sorghum) exhibit $\delta^{13} \mathrm{C}$ values ranging from $-8 \%$ to $-20 \%{ }^{14}$ In agreement with the data published recently, ${ }^{15}$ the mean $\delta^{13} \mathrm{C}$ values measured for androsterone were $-19.0 \%$, $-20.5 \%$ and $-22.0 \%$ for SAF, JAP and ITA, respectively. This trend which is also confirmed for etiocholanolone, $5 \beta$-pregnanediol and $16(5 \alpha)$-androstenol corresponds to 
Figure 1 Simplified metabolic pathway of the steroids investigated in that study.

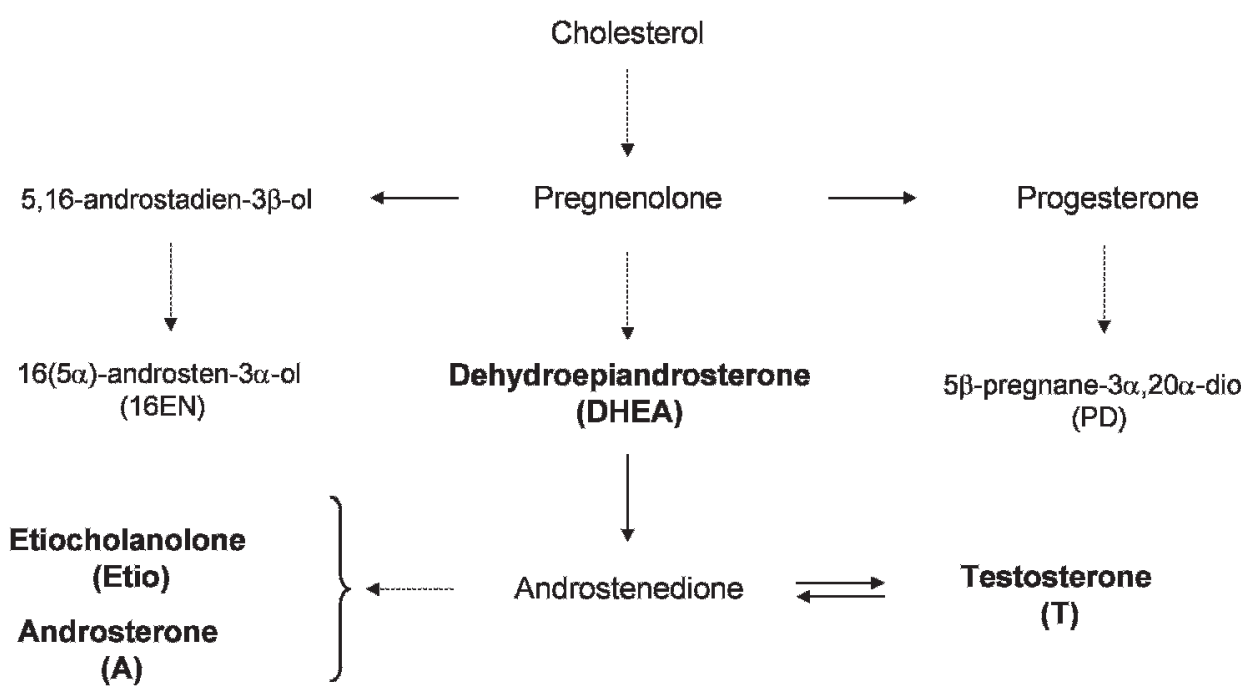

an apparent higher consumption of C3 foodstuffs by European populations compared to other countries. Depletion in ${ }^{13} \mathrm{C}$ in the steroids excreted by Italian and Swiss soccer players is reflected by more negative $\delta^{13} \mathrm{C}$ values according to the equation given previously.

It is important to note that ethnicity does not appear to contribute to variations in the ${ }^{13} \mathrm{C} /{ }^{12} \mathrm{C}$ ratio in steroids. ${ }^{15}{ }^{16}$ The natural variation in ${ }^{13} \mathrm{C} /{ }^{12} \mathrm{C}$ composition of steroids excreted in urine was recently described for men and women residing in Germany. ${ }^{17}$ For this European population, a metabolic ${ }^{13} \mathrm{C}$ fractionation was observed between androsterone and etiocholanolone. It is believed that the isotopic fractionation between $5 \alpha$ and $5 \beta$ metabolites originates from a kinetic isotope effect during the reduction step of testosterone. ${ }^{18}$ Our data show significant ${ }^{13} \mathrm{C}$ depletion in etiocholanolone compared to androsterone for ARG, JAP, ITA and SWI populations $(p<0.02)$. In contrast, both testosterone metabolites display comparable ${ }^{13} \mathrm{C}$ enrichment for the athletes of SAF and UGA.
According to WADA guidelines, a $\Delta \delta^{13} \mathrm{C}$ difference of $3.0 \%$ or more between the $\delta^{13} \mathrm{C}$ values of testosterone metabolites and endogenous reference compounds (ERC) is consistent with the administration of exogenous steroids. ${ }^{4}$ This biomarker derived from the difference between isotope ratios constitutes a corrective factor for one's individual diet. Endogenous steroids may be considered as ERC, if their carbon isotope values are not affected by the metabolism of xenobiotic compounds. Importantly, the technical document is applicable irrespective of the choice of the testosterone metabolite and the ERC chosen to calculate the $\Delta \delta^{13} \mathrm{C}$ value. In our study, it appears that the isotopic fractionation between androsterone and the ERCs is comparable for all individuals, regardless of their diet habit. Indeed, in the different groups of soccer players, no statistical difference was found between the $\Delta \delta^{13} \mathrm{C}$ values calculated from the difference between the $\delta^{13} \mathrm{C}$ values of androsterone and $16(5 \alpha)$-androstenol or $5 \beta$-pregnanediol. In agreement with these findings, Piper et al observed no significant change in the
Figure 2 Distribution of $\mathrm{d} 13 \mathrm{C}$ values obtained for etiocholanolone (Etio), androsterone (A), 16(5 2 )-androstenol (16EN) and 5 $\beta$-pregnanediol (PD) in the urine specimens of the top-level soccer players residing in the six surveyed countries.
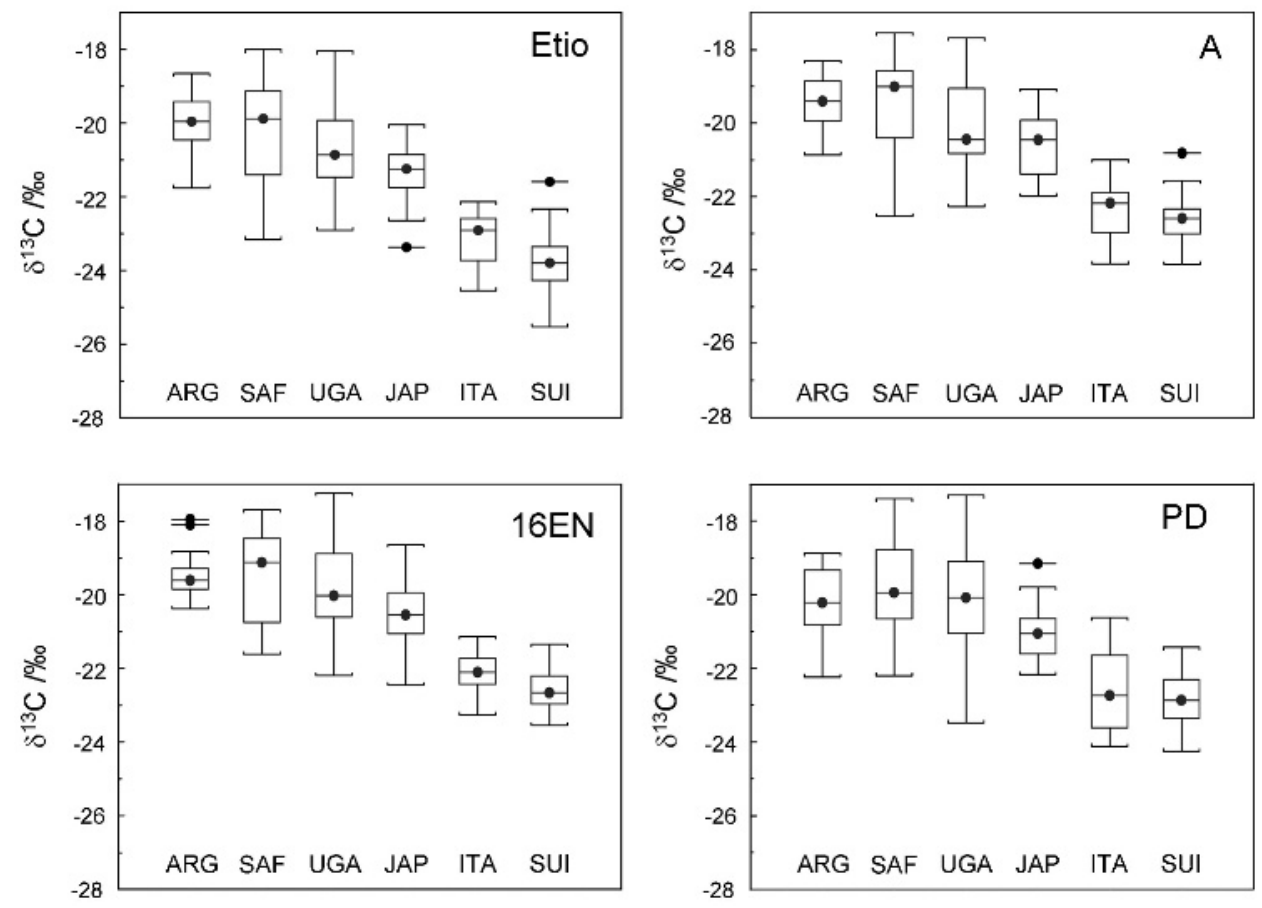
Table $199 \%$ Confidence intervals (Cls) of the $\Delta \delta^{13} \mathrm{C}$ values for each surveyed country calculated by the addition of the mean value and threefold SD

\begin{tabular}{lllll}
\hline & $\mathbf{9 9 \%} \mathbf{C l}$ & & & \\
\cline { 2 - 5 } & $\boldsymbol{\Delta}$ (16EN-Etio) & $\boldsymbol{\Delta}$ (16EN-A) & $\boldsymbol{\Delta}$ (PD-Etio) & $\boldsymbol{\Delta}$ (PD-A) \\
\hline Uganda $(\mathrm{n}=28)$ & 2.8 & 2.3 & 3.0 & 2.3 \\
South Africa $(\mathrm{n}=30)$ & 2.5 & 2.3 & 3.2 & 2.4 \\
Switzerland $(\mathrm{n}=31)$ & 3.4 & 1.2 & 3.2 & 1.4 \\
Italy $(\mathrm{n}=19)$ & 2.9 & 2.6 & 3.2 & 1.8 \\
Argentina $(\mathrm{n}=31)$ & 2.6 & 1.9 & 2.7 & 1.7 \\
Japan $(\mathrm{n}=32)$ & 3.2 & 2.9 & 2.5 & 2.3 \\
\hline
\end{tabular}

All values are in $\delta^{13} \mathrm{C}_{\mathrm{VPDB}}(\%)$.

16EN, 16(5 $\alpha)$-androstenol; A, androsterone; Etio, etiocholanolone; PD, $5 \beta$-pregnanediol.

difference between the ${ }^{13} \mathrm{C} /{ }^{12} \mathrm{C}$ ratios of $11 \beta$-hydroxyandrosterone, a product of the cortisol-cortisone metabolic pathway, and androsterone in urine samples of athletes residing in various geographical regions of the world. ${ }^{19}$

Table 1 lists the upper limit of the 99\% confidence intervals of the differences in ${ }^{13} \mathrm{C} /{ }^{12} \mathrm{C}$ composition between testosterone metabolites and the ERCs for each athletes group. These intervals are determined by the sum of the mean value and threefold the standard deviation. Our data reveal discrepancies depending on the pair of steroids that is considered, thus illustrating the occurrence of isotope fractionation in steroid metabolism. Specifically, 99\% confidence intervals for the differences in ${ }^{13} \mathrm{C} /{ }^{12} \mathrm{C}$ content between $5 \beta$-pregnanediol and androsterone are systematically lower than $2.5 \%$ whereas the $3 \%$ threshold value may not be suitable for steroid pairs based on the $\delta^{13} \mathrm{C}$ value of etiocholanolone and the ERCs.

\section{Conclusions}

IRMS is already used in several antidoping laboratories to confirm the administration of testosterone. It is expected to become a universal analytical test in the forthcoming years. Today, the differences measured between the carbon isotope ratio of testosterone or its metabolites and an endogenous reference compound are considered as evidence of doping if one value is greater that $3 \%$, the threshold set by WADA. However, to validate the assay, the intervals of a reference population must also be determined. ${ }^{20}{ }^{21}$ This study assessed the sensitivity of the assay, which is limited by the imprecision in the measurements of the carbon isotope ratio and the natural isotope fractionation occurring during the metabolism of androgens. In agreement with previous investigations, ${ }^{17}{ }^{20}$ the intervals obtained from six different world populations of soccer players clearly show that the threshold of $3 \%$ is not suitable for all the $\Delta \delta^{13} \mathrm{C}$ values determined in our study. Indeed, such a threshold is too permissive for the $\Delta \delta^{13} \mathrm{C}$ value determined from $5 \beta$-pregnanediol and androsterone, thereby decreasing the diagnostic sensitivity of the test.

Our investigation suggests that different thresholds specific for each pair of steroids should be established. Of course, the evaluation of appropriate cut-offs should be assessed though interlaboratory collaborative trials. Such an approach would significantly increase the power of the test to show testosterone misuse. To further improve the strategy in the detection of testosterone administration, urine specimens for IRMS analysis may also be selected based on the abnormal variation in steroid time profile of the athlete. ${ }^{22} 23$
Acknowledgements: This project has been carried out with support from the Fédération Internationale de Football (FIFA Medical Office: Dr Katharina Grimm and Anja Koenig). The authors are particularly indebted to the FIFA medical team: Dr Pablo Ortega Gallo and Dr Raul Madero (Argentina); Dr Demitri Constantinou and Dr Sello Motaung (South Africa); Dr Luca Gattesci and Dr Enrico Castellacci (Italy); Prof Haruhito Aoki (Japan); Dr James Sekajugo (Uganda) and Dr Roland Grossen (Switzerland). François Marclay is gratefully acknowledged for his technical assistance.

Competing interests: None declared.

Ethics approval: The study was initiated after validation of the protocol by the ethical committee of the University of Lausanne, Switzerland.

Provenance and peer review: Not commissioned; externally peer reviewed.

\section{REFERENCES}

1. Sjöqvist F, Garle M, Rane A. Use of doping agents, particularly anabolic steroids, in sports and society. Lancet 2008;31:1872-82.

2. Strahm E, Sottas PE, Schweizer C, et al. Steroid profiles of professional soccer players: an international comparative study. Br J Sports Med. Published Online First: 12 March 2009. doi:10.1136/bjsm.2008.056242

3. Saudan C, Baume N, Robinson N, et al. Testosterone and doping control. Br J Sports Med 2006; 40 (Suppl 1):i30-4.

4. WADA. WADA technical document - T2004EAAS: reporting and evaluation guidance for testosterone, epitestosterone, T/E ratio and other endogenous steroids, 2004 http://www.wada-ama.org (accessed 25 November 2008).

5. Handelsman DJ. Androgen action and pharmacologic uses. In: DeGroot LJ, Jameson JL, eds. Endocrinology. Fourth edn. Philadelphia, Pennsylvania, USA: Saunders Company, 2001: 2232.

6. Milgrom E. Steroid hormones. In: Baulieu E-E, Kelly PA, eds. Hormones. Paris, France: Hermann Publishers in Art and Science, 1990: 387.

7. Smith BN, Epstein S. Two categories of $13 \mathrm{C} / 12 \mathrm{C}$ ratios for higher plants. Plant Physiol 1971;47:380-4.

8. O'Leary MH. Carbon isotope fractionation in plants. Phytochem 1981;20:553-67.

9. DeNiro MJ, Epstein S. Influence of diet on the distribution of carbon isotopes in animals. Geochim Cosmochim Acta 1978;42:495-506.

10. WADA. The World Anti-Doping Code - The 2008 Prohibited List: International Standard, 2008. http://www.wada-ama.org (accessed 12 December 2008).

11. Saudan C, Emery C, Marclay F, et al. Validation and performance comparison of two carbon isotope ratio methods to control the misuse of androgens in humans. J Chromatogr B 2009;877:2321-9.

12. Baume N, Saudan C, Desmarchelier A, et al. Use of isotope ratio mass spectrometry to detect doping with oral testosterone undecanoate: inter-individual variability of 13C/12C ratio. Steroids 2006; $\mathbf{7 1 : 3 6 4 - 7 0 . ~}$

13. Flenker $\mathbf{U}$, von Kuk $\mathrm{C}$, Güntner $\mathrm{U}$, et al. Influence of changes in diet on the dynamics of $13 \mathrm{C} / 12 \mathrm{C}$ in selected urinary steroids: diet free from cholesterol. In: Schänzer W, Geyer H, Gotzmann A, Mareck-Engelke U, eds. Proceedings of the 23rd Cologne Workshop on Dope Analysis. Cologne, Germany: Sport und Buch Strauss, 2005: 227-33.

14. Platzner IT. Applications of isotope ratio mass spectrometry. In: Modern isotope ratio mass spectrometry. Platzner IT, ed. Chichester, UK: Wiley, 1997: 428-31.

15. Cawley AT, Trout GJ, Kazlauskas $R$, et al. Carbon isotope ratio $(\delta 13 \mathrm{C})$ values of urinary steroids for doping control in sport. Steroids 2009;74:379-92.

16. Aguilera R, Chapman TE, Starcevic B, et al. Performance characteristics of a carbon isotope ratio method for detecting doping with testosterone based on urine diols: controls and athletes with elevated testosterone/epitestosterone ratios. Clin Chem 2001;47:292-300.

17. Flenker U, Güntner U, Schänzer W. $\delta 13 \mathrm{C}$-values of endogenous urinary steroids. Steroids 2008;73:408-16.

18. Flenker U, Schänzer W. Kinetic isotope effects during metabolism of $\Delta 4$-steroids. In Schänzer W, Geyer H, Gotzmann A, Mareck-Engelke U, eds. Proceedings of the 19th Cologne Workshop on Dope Analysis. Cologne, Germany: Sport und Buch Strauss, 2001: 179-85.

19. Piper T, Flenker U, Mareck U, et al. 13C/12C Ratios of endogenous urinary steroids investigated for doping control purposes. Drug Test Analysis 2009;1:65-72.

20. Piper T, Mareck U, Geyer H, et al. Determination of $13 \mathrm{C} / 12 \mathrm{C}$ ratios of endogenous urinary steroids: method validation, reference population and application to doping control purposes Rapid Commun. Mass Spectrom 2008;22:2161-75.

21. Sottas P-E, Saudan C, Saugy M. Doping: a paradigm shift has taken place in testing Nature 2008;455:166.

22. Sottas P-E, Baume N, Saudan C, et al. Bayesian detection of abnormal values in longitudinal biomarkers with an application to T/E ratio. Biostatistics 2007:8:285-96

23. Sottas P-E, Saudan C, Schweizer C, et al. From population- to subject-based limits of T/E ratio to detect testosterone abuse in elite sports. Forensic Sci Int 2008:174:166-72. 